\title{
The Assessment of Basic Features of Electroencephalography in Metabolic Encephalopathies
}

\author{
Aylin Bican Demira, ${ }^{\mathrm{a}}$, Ibrahim Bora ${ }^{\mathrm{a}}$, Emine Kaygilia, Gokhan Ocakoglu
}

\begin{abstract}
Background: The comparison of the electroencephalography (EEG) data with the patients' primary diagnosis and the relationship with the prognosis was assessed with this study in the cases that are being followed up with the diagnosis of metabolic encephalopathy (ME).
\end{abstract}

Methods: A total of 306 patients who were being followed up due to ME between January 2009 and September 2011 were included in the study. The etiologic causes in the cases were detected as hyponatremia in $26.2 \%$, hypoxic ischemic encephalopathy in $23.8 \%$, renal failure in $14.4 \%$, hepatic failure in $11.7 \%$, diabetes mellitus in $8.2 \%$, endocrinopathies except for diabetes mellitus in $8.8 \%$, and hypernatremia in the remaining $6.9 \%$. EEG examinations were performed with two different methods. Firstly, 269 of 367 EEGs were analyzed for baseline activity, divided in six stages.

Results: Another assessment in EEG examination considering abnormal patterns was performed and 281 of 367 EEGs were taken into this assessment; reduction in the alpha, asynchronous slow waves, focal slow activities, triphasic waves, burst-suppression pattern, and generalized or focal spike-sharp activities were observed. There were no differences between the EEG groups statistically by age and sex. There were no statistical associations between diagnoses and the change of consciousness $(\mathrm{P}=0.187)$. There was no significant correlation between EEG findings and diagnostic groups ( $\mathrm{P}=0.126)$; however, it was statistically shown that as the impaired consciousness increased, the EEG stages moved forward to worse stages $(\mathrm{P}<0.001)$.

Conclusion: We think that EEG examination does not contribute

Manuscript accepted for publication August 13, 2014

${ }^{a}$ Department of Neurology, Uludag University Faculty of Medicine, Bursa, Turkey

${ }^{b}$ Department of Biostatistics, Uludag University Faculty of Medicine, Bursa, Turkey

${ }^{\mathrm{c} C}$ Corresponding Author: Aylin Bican Demir, Department of Neurology, Uludag University Medical School, Noroloji ABD, 16059 Gorukle,

Bursa, Turkey. Email: aylinbd@uludag.edu.tr

doi: http://dx.doi.org/10.14740/jnr285w to the diagnosis of the etiology of the disease; however, it may be useful in follow-ups and prognosis in ME.

Keywords: Metabolic encephalopathy; Diagnosis; EEG; Burstsuppression pattern; Triphasic wave

\section{Introduction}

Normal brain function is dependent on the normal neuronal metabolism which is associated with the systemic homeostasis of the metabolites such as glucose, electrolytes, amino acids and ammonia. As a result of failure of organ systems such as kidneys, liver, lungs and the respiratory system, heart and the circulatory system, a general deterioration of the brain function occurs. The secondary impairment of the brain from the failures of these systems is called "metabolic encephalopathy" (ME). Ischemic-hypoxic encephalopathy that occurs as a result of the cessation or reduction of the oxygen or the blood flow reaching the brain is one of the acquired MEs of the nerve system. The most common medical conditions that cause this condition include heart attack, ventricular arrhythmia, blood loss, decrease in cerebral blood flow due to septic or traumatic shock, drowning, vomiting, foreign body obstruction in the trachea, the carbon monoxide poisoning, toxications, Guillain-Barre syndrome, amyotrophic lateral sclerosis, diseases which cause paralysis of the respiratory muscles such as myasthenia gravis, diseases that cause extensive central nervous system damage particularly involving the medulla, insufficient oxygen in the patient's respiratory gas during general anesthesia, diseases of the organs other than the brain (non-endocrine organ diseases, hyper and hypofunctions of the endocrin organs, water and electrolyte imbalance), hyperthermia, acidosis, hypoglycemia, and hypercapnia [1-3].

Change in consciousness is one of the clinical symptoms that is often encountered in emergency situations and requires rapid diagnosis and treatment. In this case, electroencephalography (EEG) is one of the required tests in order to diagnose and partially get an idea of the prognosis. An EEG should be performed in the differential diagnosis of whether 


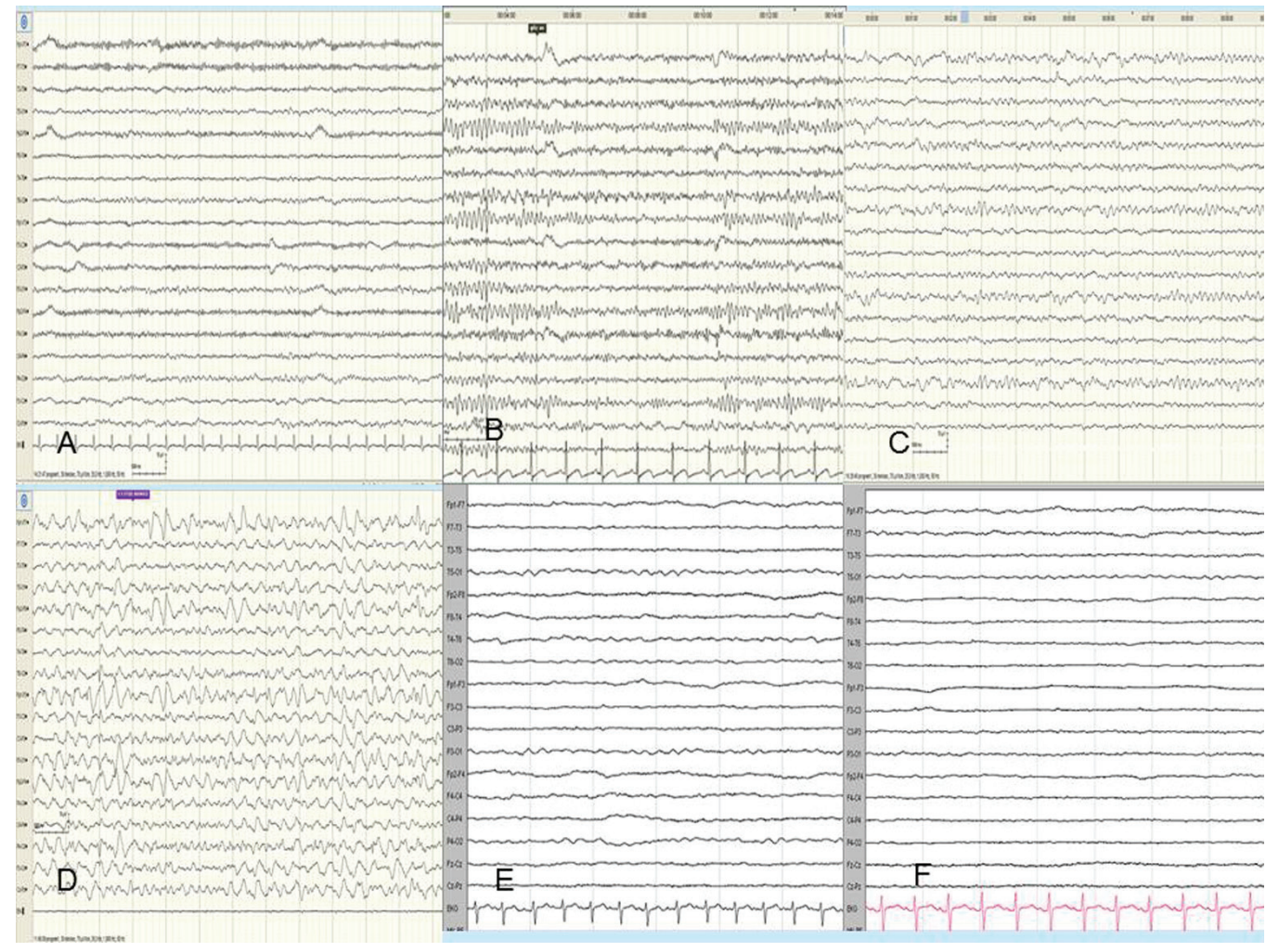

Figure 1. (A) Normal ground rhythm. (B) Decreased alpha activity that reacts to opening and closing of the eye and the recordings with partially increased tetha waves. (C) Recordings that show locally observed alpha activity that does not react to opening and closing of the eye, extensive tetha activity and sometimes brief paroxysms of concomitant delta activity. (D) Ground activity with extensive high-amplitude delta activity. (E) Ground rhythm with low-amplitude delta activity. (F) Isoelectric EEG recording.

the change in consciousness, transient loss of consciousness or an abnormal motor movement is caused from an epileptic activity. In emergency situations, an EEG is usually required in conditions such as status epilepticus, encephalitis and delirium. An EEG may be performed also in patients that already have a diagnosis to organize the treatment and get an idea about the prognosis.

In 1937, during Berger's treatment of schizophrenic patients with insulin, with the determination of the deceleration in the rhythm of the ground in EEG after the activated hypoglycemia, EEG in ME was identified as one of the important examinations in order to assess the clinical condition and the prognosis [4].

In our study, the comparison of the EEG data and patients' primary diagnosis and their association in terms of the prognosis in patients with the diagnosis of ME is evaluated.

\section{Methods}

Patients were first hospitalized mostly by departments other than neurology (nephrology, endocrinology, gastroenterology, cardiology, reanimation unit, emergency room, etc.) with the diagnosis of confusion, they were included in the study after they were consulted by neurology and their EEG examinations were performed with a provisional diagnosis of ME. A total of 367 EEGs of 306 patients were performed with a 21-channel EEG (device brand Medelek) by the placements of the superficial scalp electrodes according to the International 10-20 system. EEG examinations of the patients were evaluated retrospectively. Metabolic charts and treatment protocols of the patients have been reached from the hospital records of the patients at the time of admission.

The demographic data, biochemical values, the neurological examinations that were stated in the neurology consultation, primary diseases, concomitant systemic diseases, imaging results and the EEG data of the patients were evaluated. EEG data were evaluated and reported by an experienced epileptologist.

EEG was analyzed by two different methods. First one was based on the basic activity whereas the second one was performed considering the abnormal patterns [5]. 1) Stage 1: Normal ground rhythm (Fig. 1A). 2) Stage 2: Decreased alpha activity that reacts to opening and closing of the eye and the recordings with partially increased tetha waves (Fig. 1B). 3) Stage 3: Recordings that show locally observed alpha 


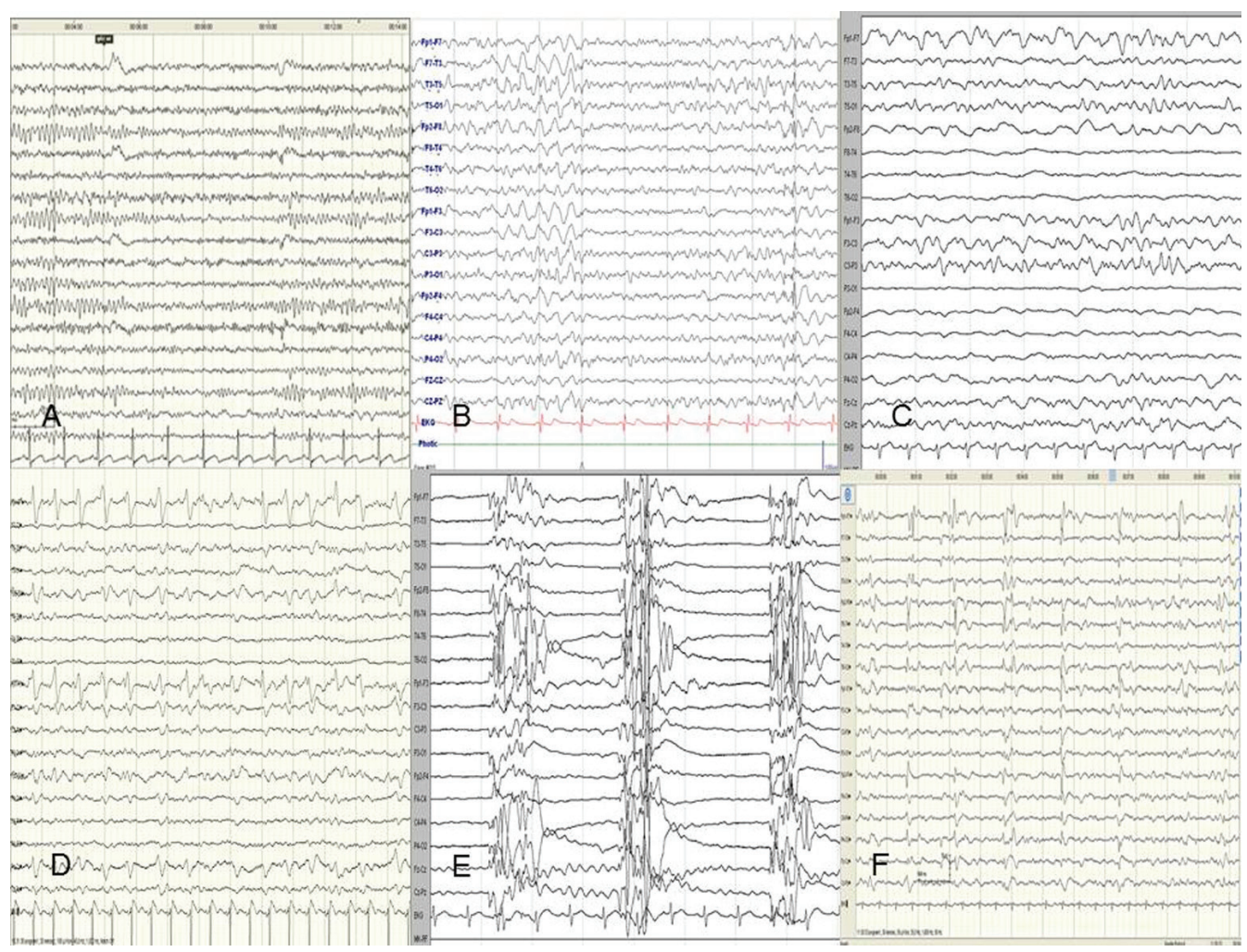

Figure 2. (A) Decrease in alpha frequency. (B) Generalized asynchronous slow waves. (C) Focal slow activities. (D) Triphasic waves. (E) Burst-suppression pattern. (F) Generalized or focal spike-sharp activities.

activity that does not react to opening and closing of the eye, extensive tetha activity and sometimes brief paroxysms of concomitant delta activity (Fig. 1C). 4) Stage 4: Ground activity with extensive high-amplitude delta activity (Fig. 1D). 5) Stage 5: Ground rhythm with low-amplitude delta activity (Fig. 1E). 6) Stage 6: Isoelectric EEG recording (Fig. 1F).

Another assessment of the EEG examination is classified as decrease in alpha frequency (Fig. 2A), generalized asynchronous slow waves (Fig. 2B), focal slow activities (Fig. 2C), triphasic waves (Fig. 2D), burst suppression pattern (Fig. 2E), and generalized or focal spike-sharp activities (Fig. 2F).

\section{Statistical findings}

Age and EEG variables were expressed as the descriptive statistics by their median (minimum-maximum) values, whereas the categorical variables were expressed by their number and relative percentage in the study. Kruskal-Wallis and Mann-Whitney tests were performed while comparing the age values and the EEG scores between the groups and Chi-square test was used while comparing the categorical variables between the groups. The analyses of the study were made using SPSS 13.0 statistical analysis software and $\mathrm{P}<$ 0.05 was considered statistically significant.

\section{Results}

Of the patients, $52.6 \%$ were male, $47.4 \%$ were female and their median age was 63 years (19-88 years). Depending on the age groups and genders and the etiological causes of our study group, there was not a difference between the distribution of the diagnosis of the diseases and it was a homogenous grouping (Table 1).

The etiologic causes in the cases were most commonly hyponatremia in $26.2 \%$, second most commonly hypoxic ischemic encephalopathy (HIE) in $23.8 \%$, renal failure in $14.4 \%$, hepatic failure in $11.7 \%$, diabetes mellitus in $8.2 \%$, endocrinopathies except for diabetes mellitus (hyper-hypoparathyrodisim, Addison's, thyrotoxicosis) in $8.8 \%$, and hypernatremia in the remaining $6.9 \%$ (Table 2).

Looking at the systemic diseases associated with the cases, hypertension in $23 \%$, heart diseases in $19 \%$, diabetes mellitus in $14 \%$, previous brain vascular disease in $12 \%$, hyperlipidemia in $9 \%$, dementia in $3 \%$, anemia in $3 \%$ and the remaining $17 \%$ was present with more than one coexisting disease.

According to the state of consciousness, it was determined that $9.8 \%$ were conscious, $32.7 \%$ had confusion, $26 \%$ were lethargic, $18.4 \%$ were in stupor, and $13.1 \%$ were at the level of coma (Table 3). 
Table 1. Demographic Results

\begin{tabular}{llll}
\hline Diagnostic subgroups & Woman & Man & Age \\
\hline Hyponatremia $(\mathrm{n}=80)$ & $44(11.4)$ & $36(11.8)$ & $68(21-77)$ \\
Hypoxic $(\mathrm{n}=73)$ & $24(7.8)$ & $49(16)$ & $66(30-76)$ \\
Renal failure $(\mathrm{n}=44)$ & $17(5.6)$ & $27(8.8)$ & $58(23-69)$ \\
Hepatic insufficiency $(\mathrm{n}=36)$ & $16(5.2)$ & $20(6.5)$ & $56(19-67)$ \\
Diabetes mellitus $(\mathrm{n}=25)$ & $14(4.6)$ & $11(3.6)$ & $67(20-80)$ \\
Endocrinopathy $(\mathrm{n}=27)$ & $20(6.5)$ & $7(2.3)$ & $54(19-66)$ \\
Hypernatremia $(\mathrm{n}=21)$ & $10(3.3)$ & $11(3.6)$ & $69(26-88)$ \\
\hline
\end{tabular}

Data are shown as $\mathrm{n}(\%)$ and median (minimum-maximum).

In the results of the EEG examinations, 269 of 367 EEGs were evaluated in terms of basic activity: stage 1,6\%; stage $2,37 \%$; stage 3, 24\%; stage 4, 23\%; stage 5, 6\%; stage 6, 4\% (Table 4).

Another assessment in EEG examination considering abnormal patterns was performed and 281 of 367 EEGs were taken into this assessment; reduction in the alpha frequency in $30 \%$, generalized asynchronous slow waves in $36.6 \%$, fo- cal slow activities in $12.3 \%$, triphasic waves in $6.4 \%$, burstsuppression pattern in $1 \%$, and generalized or focal spikesharp activities in $2.6 \%$ were observed (Table 5).

There were no differences between the EEG groups statistically by age and sex $(\mathrm{P}=0.263$ and $\mathrm{P}=0.841)$. There were no statistical associations between diagnoses and the change of consciousness $(\mathrm{P}=0.187)$. There was no significant correlation between EEG findings and diagnostic

Table 2. Disease Groups and the Level of Consciousness

\begin{tabular}{llllll}
\hline Diagnostic subgroups & Normal & Confused & Lethargic & Stupor & Coma \\
\hline Hyponatremia $(\mathrm{n}=80)$ & $8(2.6)$ & $22(7.2)$ & $35(11.4)$ & $12(3.9)$ & $3(1)$ \\
Hypoxic $(\mathrm{n}=73)$ & $0(0)$ & $31(10.1)$ & $20(6.5)$ & $11(3.6)$ & $11(3.6)$ \\
Renal failure $(\mathrm{n}=44)$ & $14(4.6)$ & $13(4.2)$ & $9(2.9)$ & $6(2)$ & $2(0.7)$ \\
Hepatic insufficiency $(\mathrm{n}=36)$ & $0(0)$ & $3(1)$ & $3(1)$ & $16(5.2)$ & $14(4.6)$ \\
Diabetes mellitus $(\mathrm{n}=25)$ & $4(1.3)$ & $11(3.6)$ & $4(1.3)$ & $2(0.7)$ & $4(1.3)$ \\
Endocrinopathy $(\mathrm{n}=27)$ & $4(1.3)$ & $10(3.3)$ & $4(1.3)$ & $5(1.6)$ & $4(1.3)$ \\
Hypernatremia $(\mathrm{n}=21)$ & $0(0)$ & $10(3.3)$ & $5(1.6)$ & $4(1.3)$ & $2(0.7)$ \\
\hline
\end{tabular}

Data are shown as $\mathrm{n}(\%)$. 
Table 3. Disease Groups and EEG Findings, According to the Basic Activity

\begin{tabular}{lllllll}
\hline Diagnostic subgroups & Stage 1 & Stage 2 & Stage 3 & Stage 4 & Stage 5 & Stage 6 \\
\hline Hyponatremia $(\mathrm{n}=80)$ & $6(2)$ & $11(3.6)$ & $31(10.1)$ & $10(3.3)$ & $3(1)$ & $1(0.3)$ \\
Hypoxic $(\mathrm{n}=73)$ & $5(1.6)$ & $23(7.5)$ & $13(4.2)$ & $9(2.9)$ & $4(1.3)$ & $4(1.3)$ \\
Renal failure $(\mathrm{n}=44)$ & $5(1.6)$ & $20(6.5)$ & $10(3.3)$ & $8(2.6)$ & $1(0.3)$ & $0(0)$ \\
Hepatic insufficiency $(\mathrm{n}=36)$ & $0(0)$ & $1(0.3)$ & $4(1.3)$ & $20(6.5)$ & $5(1.6)$ & $6(2)$ \\
Diabetes mellitus $(\mathrm{n}=25)$ & $0(0)$ & $24(7.8)$ & $0(0)$ & $3(1)$ & $0(0)$ & $0(0)$ \\
Endocrinopathy $(\mathrm{n}=27)$ & $0(0)$ & $10(3.3)$ & $3(1)$ & $6(2)$ & $2(0.7)$ & $0(0)$ \\
Hypernatremia $(\mathrm{n}=21)$ & $0(0)$ & $10(3.3)$ & $4(1.3)$ & $6(2)$ & $1(0.3)$ & $0(0)$ \\
\hline
\end{tabular}

Data are shown as $\mathrm{n}(\%)$.

groups ( $\mathrm{P}=0.126)$; however, it was statistically shown that as the impaired consciousness increased, the EEG stages moved forward to worse stages ( $\mathrm{P}<0.001)$.

\section{Discussion}

ME is the secondary impairment of the brain as a result of a general deterioration of the brain function caused by variable organ system failures such as kidneys, liver, lungs and the respiratory system, heart and the circulatory system without primary structural abnormalities of the central nervous system [1-3].

The electrical membrane potential of a nerve cell is provided by the energy-requiring work of Na-K ATPase and similar ion pumps. The metabolic fuel in the brain in normal circumstances is glucose. However, only in the fasting state ketone bodies instead of glucose are used as a fuel. Daily glucose need of the brain is approximately $120 \mathrm{~g}$. It forms $15 \%$ of daily total energy consumption. Oxygen is needed in order to use glucose as a fuel or for the oxidation of glucose. Twenty percent of total oxygen consumption in the body takes place in the brain and $60 \%$ of it is used in ATP synthesis for electrical activity. EEG is based on the principle of the recording of the fluctuation in the electrical activity of a large group of neurons in the brain.

Most of the potentials that are recorded from the scalp are the results of the relationship of total sinaptic potentials in pyramidal cells with extra-cellular currents. Even though the main use of EEG is to evaluate epilepsy patients, another patient group that EEG is very important and essential for is the emergency cases that are likely to be encephalitis or encephalopathy [6].

Khan et al emphasized that EEG gives the best information about particularly indications associated with epilepsy, hypoxic encephalopathy and brain death cases; however, it gives less information about encephalitis, acute disseminated encephalomyelitis, suspected or ME cases [7].

In our study, hyponatremic encephalopathy accounted for $26.3 \%(n=80 / 306)$ of the ME patient group. It was detected that 47 of these cases had isolated hyponatremia, 13 developed hyponatremia due to chronic kidney disease, and 30 due to diabetes. In these cases hyponatremia was the main finding and the current disease was stabile. Main abnormality in our cases was that the EEG findings of these cases were electrophysiological findings that are related to combined metabolic disorders, because hyponatremia was perched on the existing metabolic disorder. In our study, according to the state of consciousness, in 80 hyponatremic cases $7.2 \%$ $(\mathrm{n}=22)$ had confusion and $11.4 \%(\mathrm{n}=35)$ were letharjic. In the examination according to EEG ground activity, we observed $10.1 \%(n=31)$ of stage 3 recordings that show locally observed alpha activity that does not react to opening and closing of the eye, extensive tetha activity and sometimes brief paroxysms of concomitant delta activity. In the EEG examination according to pathological activity, decrease in alpha activity was $7.8 \%(\mathrm{n}=24)$ and generalized asynchronous slow wave activity was $10 \%(\mathrm{n}=32)$.

In an article of Kaplan's it is mentioned that in the EEG studies that were performed in the patients that had encephalopathy due to hyponatremia, decreases in the alpha activity and intensive delta activity were present and we had similar findings in our study [5].

The earliest change in anoxia is seen in the first $30 \mathrm{~min}$, 


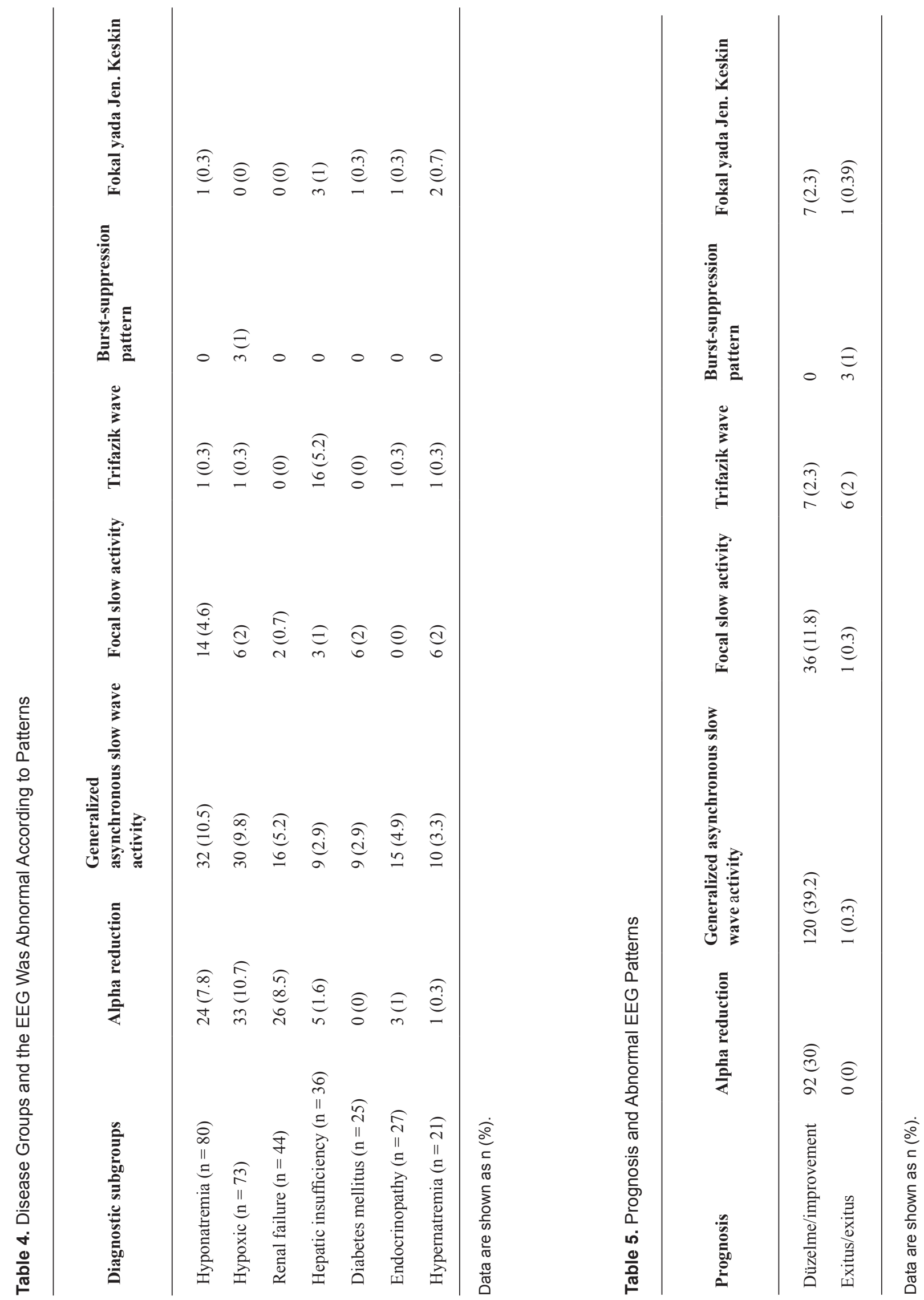


Table 6. Prognosis and EEG Investigations, According to the Basic Activity

\begin{tabular}{lllllll}
\hline Prognosis & Stage 1 & Stage 2 & Stage 3 & Stage 4 & Stage 5 & Stage 6 \\
\hline Improvement & $16(5.2)$ & $99(32.4)$ & $64(20.9)$ & $60(19.6)$ & $11(3.6)$ & $7(2.3)$ \\
Exitus & $0(0)$ & $0(0)$ & $1(0.3)$ & $2(0.7)$ & $5(1.6)$ & $4(1.3)$ \\
\hline
\end{tabular}

Data are shown as $\mathrm{n}(\%)$.

in the electrochemical environment where intracellular and extracellular acidification is observed. The more severe anoxia is, the more severe the electrophysiological findings are and the later the EEG findings improve. In parallel, the pathological damage is that serious. As a result of cardiopulmonary arrest, the most common pattern in EEG is $\alpha$ (alpha) in the first day; however, extensive or slow wave activity, triphasic wave may accompany in different rates, and the developing burst-suppression pattern is related to bad prognosis and associated with severe neuronal damage [8].

In our study, 24\% (73/306) of the cases had a diagnosis of HIE. Different causes (myocardial infarction, heart failure, hanging, drowning post-operative cardiac arrest, and cardiac arrest due to complete AV block) were detected in the etiology of HIE.

Patients' level of cognition was found as confusion in $10.1 \%(\mathrm{n}=31)$, lethargic in $6.5 \%(\mathrm{n}=20)$, and stupor and coma in $3.6 \%(\mathrm{n}=11)$. According to the examination based on EEG ground activity stage 2 was found in $7.5 \%(n=23)$ and stage 3 was found in $4.2 \%(n=13)$ and we observed recordings with decreased alpha activity with partially increases in tetha waves and recordings that show locally observed alpha activity that does not react to opening and closing of the eye, extensive tetha activity and sometimes brief paroxysms of concomitant delta activity. In the EEG examination according to pathological activity, we observed decrease in alpha activity in $10.7 \%(\mathrm{n}=33)$, generalized asynchronous slow wave activity in $9.8 \%(\mathrm{n}=30)$, and burst-suppression pattern in $1 \%(n=3)$. Three cases with the burst-suppression pattern died $(100 \%, 3 / 3)$.

Of the cases $23.3 \%(n=17)$ that had compatible findings in EEG stages 4-6 were diagnosed as HIE. Of the cases $65 \%(11 / 17)$ that were being followed-up with a diagnosis of HIE recovered; however, it was concluded that the remaining $35 \%(6 / 17)$ died.

In anoxic events, in order to determine irreversible neuropathological and functional damage, the duration and severity of the abnormality of EEG is more sensitive than measurable cerebral blood flow or energy metabolism [8]. Therefore, in the cases diagnosed with HIE, EEG examination had an important role in order to determine the progno- sis as in our study group.

Uremic encephalopathy is one of the organic brain syndromes that develop in some patients with chronic renal failure or who have a glomerular filtration rate less than $10 \%$. It may have a large neurologic symptom spectrum starting with fluctuation on consciousness [9].

In our study, $14.4 \%(\mathrm{n}=44)$ of the patients were diagnosed as uremic encephalopathy; the cognition levels were open in $4.6 \%(n=14)$, confused in $4.2 \%(n=13)$ and lethargic in $2.9 \%(n=9)$ and in the EEG examinations $6.5 \%(n$ $=20)$ of them were observed as stage 2 , and $3.3 \%(n=10)$ were observed as stage 3 . In the results in terms of EEG's abnormal patterns, decrease in alpha activity was observed in $8.5 \%(n=26)$ and generalized asynchronous slow wave activity was observed in $5.2 \%(\mathrm{n}=16)$. In our study there were no focal or sharp generalized activity or triphasic waves. EEG abnormalities can be recorded $48 \mathrm{~h}$ after the symptoms of renal failure. Slow activities and/or epileptiform changes that are usually localized in frontal regions are detected in $90 \%$ of abnormal EEG examinations. Furthermore, bilateral spike wave activity can be detected in $14 \%$ of the patients who clinically do not have seizures and in $20 \%$ triphasic waves can be encountered in direct proportion of blood urea level. Although the azotemia level is limitedly associated with encephalopathy, increasing of some electrolytes (blood urea nitrogen, creatine, potassium, chloride, and sodium) or decreasing (calcium, albumin, hematocrite, and carbon dioxide) shows a direct proportional correlation with EEG abnormality. Uysal et al did not find any correlation between the electrolyte levels and EEG abnormalities [9].

Hepatic encephalopathy (HE) which is one of the other causes of $\mathrm{ME}$, is a clinical condition that is reversible, occurs as a result of metabolic, neurophysiological disorder of the brain, and has mental, neuromuscular dysfunction findings. Clinical findings in HE develop insidiously and progress rapidly. There is only a few specific tests to determine encephalopathy and one of them is EEG [10].

As the clinical symptoms develop and ammonia level increases, deterioration in accordance with the stages occurs. Triphasic waves may appear usually in metabolic, non-specific, also sometimes in other neurologic conditions. Cases 
that have this finding are known to have a mortality of $50 \%$ in the first 30 days [11].

The purpose in HE which has a very broad clinical and electrophysiological spectrum is to determine encephalopathy which is subclinical and take precautions. In our study, stupor was observed in 5.2\% $(\mathrm{n}=16)$ and coma in $4.6 \%(\mathrm{n}$ $=14$ ) which is a high rate in the cognitive conditions of the cases which took our attention. Triphasic waves were observed in 16 of the 36 cases $(5.2 \%)$ with chronic liver disease in our study. EEG abnormalities of the cases were severe and they were observed as stage 3-6, predominantly stage 4 in $6.5 \%(n=20 / 36)$. Furthermore, four cases out of six $(66.7 \%)$ that had triphasic waves died. Six of the 31 cases $(19.4 \%)$ that were stage 4-6 died.

EEG abnormalities may be determined in $25-75 \%$ of diabetic cases. Persistent EEG abnormalities are seen in 31$80 \%$ of the cases that have a history of recurrent hypoglycemia [12]. Deceleration in EEG is usually seen on the anterior regions of the brain and it is considered that these regions are more sensitive to hypoglycemia. The most sensitive parameter in EEG abnormalities that are seen in hypoglycemic episodes is the alpha/tetha rate [13]. In our study, 9.3\% (n =25) of the cases developed ME due to hyperglycemia or hypoglycemia. As in all ME cases, the main complaint was impaired consciousness in all of the diabetic patients. However, in $3.6 \%(\mathrm{n}=11)$ of these cases there were patients from confusion to open conscious and coma. Furthermore, stage 2 findings were seen in only $7.8 \%(n=24)$ of the cases. In the staging of EEG according to abnormal patterns, generalized asynchronous slow wave activity in $2.9 \%(n=9)$, focal slow wave activity in $2 \%(n=6)$ and focal or generalized sharp wave activity in one case were detected.

The clinical findings of endocrinopathies appear depending on the hormone's individual affect or combined individuality such as 1) the changes of whole body electrolyte or substrate environment, 2) the effect (or deficiency) of the hormone on brain's metabolic electrical activity, and 3) acting of the hormone as a neurotransmitter [8].

Although the triphasic waves are seen in $9-20 \%$ of the MEs that are caused by azotemia, it has been reported very rarely in association with hyperthyroidism and therefore it may be seen in myxedema coma [14].

No correlation was found between hormonal level and electrophysiological parameters in many studies [15].

The determined encephalopathy cases that are secondary to endocrine diseases in our study group were $8.8 \%$ (n = 27 ) and their level of consciousness was normal in $1.3 \%$ (n $=4)$, confused in $3.3 \%(\mathrm{n}=10)$, lethargic in $1.3 \%(\mathrm{n}=4)$, stupor in $1.6 \%(\mathrm{n}=5)$ and $1.3 \%(\mathrm{n}=4)$ in coma. Although there were compatible EEG findings with the level of consciousness in this group, it was learned that all of the cases were discharged with recovery. Furthermore, only $3.3 \%$ (n = 10) of the cases had stage 2 finding. In the staging of EEG according to abnormal patterns, generalized asynchronous slow wave activity was detected in $4.9 \%(\mathrm{n}=15)$.

Hypernatremia is a rarely seen cause of electrolyte disorder encephalopathy and the level of consciousness may be seen from confusion to coma. High-amplitude delta activities, rarely triphasic waves and epileptiform discharges may be seen in EEG [5]. In our study, 6.9\% $(n=21)$ of encephalopathy cases due to hypernatremia, level of consciousness were, confused in 3.3\% $(\mathrm{n}=10)$, lethargic in $1.6 \%(\mathrm{n}=5)$, stupor in $1.3 \%(n=4)$, coma in $0.7 \%(n=2)$ and in the EEG examinations stage 2 in $3.3 \%(n=10)$, stage 3 in $1.3 \%(n=$ $4)$, stage 4 in $2 \%(n=6)$, stage 5 in $0.7(n=2)$ was observed. In the results that we evaluated according to EEG's abnormal patterns, decrease in alpha activity in $0.3 \%(\mathrm{n}=1)$, generalized asynchronous slow wave in $3.3 \%(\mathrm{n}=10)$, focal slow activity in $2 \%(n=6)$, triphasic activity in $0.3 \%(n=1)$, and focal or generalized sharp activity in $0.7 \%(n=2)$ was observed.

The EEG changes in acute phase are known to be the same in hypoglycemic episode and hypoxic ischemic period, but it should not be forgotten that the electrophysiological findings are nonspecific regarding $\mathrm{ME}$; however, it is a useful non-invasive examination in determining the patients' clinical conditions and prognosis. Some specific EEG findings (burst-suppression pattern, generalized suppression and alpha tetha coma) were found associated with bad prognosis [7]. In our study, $1 \%(\mathrm{n}=3)$ of burst-suppression activity was seen in hypoxic conditions and all of the cases have died. In our study triphasic waves were seen in 16 out of 36 $(5.2 \%)$ cases with chronic liver disease. EEG abnormalities of the cases were severe and it was observed as stage 3-6, predominantly stage 4 in $6.5 \%(n=20 / 36)$. Four cases out of six $(66.7 \%)$ that had triphasic waves died. Six of the 31 cases $(19.4 \%)$ that was stage 4-6 died. However it is considered as a disadvantage that EEG does not give enough information about brain stem functions. These disadvantages are partially reduced by further imaging processes [16].

There were no correlation between diagnosis subgroups and EEG in cases with ME (Table 3, 4); however, we determined a statistically significant correlation between the level of consciousness and diagnosis subgroups (Table 2) and more importantly between EEG and prognosis (Table 6).

\section{Significance of EEG in terms of prognosis}

EEG is a non-invasive test that is easily applicable and shows brain functions. Its prognostic value is greater when the reason for the change in consciousness is known. In a summary of the good and bad prognosis from EEG in our study, good prognostic factors are: 1) In EEG's classification of abnormal patterns, $94.6 \%$ of the cases that had decrease in alpha, generalized asynchronous slow wave activity, focal slow activity recovered clinically. 2) In EEG's classification of basic activity $93 \%$ of the cases that were stage 1-4 recovered. Bad prognostic factors are: 1) The cases that had 
burst-suppression pattern in EEG had hypoxia in etiology and $100 \%$ of them died. 2) Thirty-five percent of the cases with triphasic wave patterns died. 3) In EEG's classification of basic activity, it was observed that $75 \%$ of the cases that were stage 5, 6 died.

\section{Result}

EEG is important for the evaluation of clinical progression and follow-up of the patients with a suspection of convulsive and/or nonconvulsive status epilepticus and with a diagnosis of HIE and HE. As a result of this study, we determined that EEG is not appropriate for every patient who has a history of impaired consciousness; it is proper to do the EEG examination in the suspected cases that the neurological examination and clinical information are combined.

\section{References}

1. Stevens RD, Pronovost PJ. The spectrum of encephalopathy in critical illness. Semin Neurol. 2006;26(4):440451.

2. Stevens RD, Nyquist PA. Types of brain dysfunction in critical illness. Neurol Clin. 2008;26(2):469-486, ix.

3. Angel MJ, Young GB. Metabolic encephalopathies. Neurol Clin. 2011;29(4):837-882.

4. Niedermeyer E. Metabolic central nervous system disorders. In: Electroencephalography basic principles, clinical applications and related fields, Niedermeyer E, Lopes Da Silva F. Eds. Lippcott Williams and Wilkins, Maryland. Fourth edition, 1999;416-431.

5. Kaplan PW. The EEG in metabolic encephalopathy and coma. J Clin Neurophysiol. 2004;21(5):307-318.

6. Paulson OB, Hasselbalch SG, Rostrup E, Knudsen GM,
Pelligrino D. Cerebral blood flow response to functional activation. J Cereb Blood Flow Metab. 2010;30(1):2-14.

7. Firosh Khan S, Ashalatha R, Thomas SV, Sarma PS. Emergent EEG is helpful in neurology critical care practice. Clin Neurophysiol. 2005;116(10):2454-2459.

8. van Putten MJ, van Putten MH. Uncommon EEG burstsuppression in severe postanoxic encephalopathy. Clin Neurophysiol. 2010;121(8):1213-1219.

9. Uysal S, Renda Y, Saatci U, Yalaz K. Neurologic complications in chronic renal failure: a retrospective study. Clin Pediatr (Phila). 1990;29(9):510-514.

10. Reeves RR, Struve FA, Burke RS. Quantitative EEG analysis before and after liver transplantation. Clin EEG Neurosci. 2006;37(1):34-40.

11. Bahamon-Dussan JE, Celesia GG, Grigg-Damberger MM. Prognostic Significiance of EEG Triphasic Waves in Patients with Altered State of Consciousness. J Clin Neurophysiolgy. 1989;4:313-319.

12. Bendtson I, Gade J, Rosenfalck AM, Thomsen CE, Wildschiodtz G, Binder C. Nocturnal electroencephalogram registrations in type 1 (insulin-dependent) diabetic patients with hypoglycaemia. Diabetologia. 1991;34(10):750-756.

13. Asvold BO, Sand T, Hestad KA, Bjorgaas MR. Quantitative EEG in type 1 diabetic adults with childhood exposure to severe hypoglycaemia: a 16 year follow-up study. Diabetologia. 2011;54(9):2404-2408.

14. River Y, Zelig O. Triphasic waves in myxedema coma. Clin Electroencephalogr. 1993;24(3):146-150.

15. Khedr EM, El Toony LF, Tarkhan MN, Abdella G. Peripheral and central nervous system alterations in hypothyroidism: electrophysiological findings. Neuropsychobiology. 2000;41(2):88-94.

16. Praline J, Grujic J, Corcia P, Lucas B, Hommet C, Autret A, de Toffol B. Emergent EEG in clinical practice. Clin Neurophysiol. 2007;118(10):2149-2155. 